\title{
miR-181b-5p inhibits trophoblast cell migration and invasion through targeting S1PR1 in multiple abnormal trophoblast invasion-related events
}

\author{
JUAN MIAO $^{1 *}$, YIQING ZHU ${ }^{2 *}$, LEI XU ${ }^{1}$, XIAOHAO HUANG ${ }^{1}$ and XUE ZHOU ${ }^{2}$ \\ ${ }^{1}$ Department of Gynecology, The First Affiliated Hospital of Nanjing Medical University, Nanjing, \\ Jiangsu 210000; ${ }^{2}$ Department of Obstetrics, Women's Hospital of Nanjing Medical University, \\ Nanjing Maternity and Child Health Care Hospital, Nanjing, Jiangsu 210004, P.R. China
}

Received November 17, 2019; Accepted August 6, 2020

DOI: $10.3892 / \mathrm{mmr} .2020 .11515$

\begin{abstract}
Normal placentation and successful maintenance of pregnancy depend on the successful migration and invasion of trophoblasts into maternal tissues. Previous studies reported that microRNAs (miRs) are expressed in trophoblasts, and can regulate their migration and invasion. The present study aimed to investigate miR-181b-5p function in HTR-8/SVneo trophoblasts and explore its underlying mechanism in the pathogenesis of multiple abnormal trophoblast invasion-related events. Reverse-transcription quantitative PCR and western blotting were used to test the expression of miR-181b-5p and sphingosine-1-phosphate receptor 1 (S1PR1) in samples of multiple abnormal trophoblast invasion-related events. Transwell invasion and wound healing assays were performed to determine cell invasion and migration abilities. A luciferase reporter assay was conducted to identify the downstream target of miR-181b-5p. Overexpression of miR-181b-5p suppressed HTR-8/SVneo cell migration and invasion, whereas inhibition of miR-181b-5p induced an opposite effect. The S1PR1 gene was further identified as a
\end{abstract}

Correspondence to: Dr Xue Zhou, Department of Obstetrics, Women's Hospital of Nanjing Medical University, Nanjing Maternity and Child Health Care Hospital, 123 Tianfeixiang, Mochou Road, Nanjing, Jiangsu 210004, P.R. China

E-mail: maggiezhouxue840@126.com

Dr Xiaohao Huang, Department of Gynecology, The First Affiliated Hospital of Nanjing Medical University, 300 Guangzhou Road, Nanjing, Jiangsu 210000, P.R. China

E-mail: xiaohao512006@126.com

*Contributed equally

Abbreviations: SA, spontaneous abortion; $\mathrm{CH}$, choriocarcinoma; $\mathrm{NV}$, normal early pregnancy; NP, normal full-term pregnancy

Key words: microRNA-181b-5p, trophoblasts, migration, invasion, sphingosine-1-phosphate receptor 1 novel direct target of miR-181b-5p. Specifically, miR-181b-5p bound directly to the 3'-untranslated region of S1PR1 and suppressed its expression. Moreover, overexpression of S1PR1 reversed the inhibitory effect of miR-181b-5p. Taken together, ectopic expression of miR-181b-5p impaired the migration and invasion of trophoblasts by directly targeting S1PR1, thereby providing new insights into the pathogenesis of multiple abnormal trophoblast invasion-related events.

\section{Introduction}

Trophoblast migration and invasion are proposed to play a pivotal role in the process of embryo implantation and placentation, which are critical for embryonic development and successful pregnancy (1). Numerous similarities exist between embryo implantation and the growth of cancer cells; however, the former is stringently controlled both spatially and temporally $(2,3)$. The invasion of trophoblasts to a sufficient depth of the uterus is a key factor in determining the outcome of pregnancy (1). Dysregulation of this invasive behavior can trigger a wide spectrum of pregnancy-related diseases which can occur throughout various stages of pregnancy (4). Inadequate invasion can lead to pathological pregnancies such as infertility (5), miscarriage (6-8), premature birth (5), fetal growth restriction $(5,7)$ and preeclampsia $(\mathrm{PE})(5,7,9)$, while excessive invasion has been implicated in the pathophysiology of placenta accreta $(1,5)$ and premalignant or malignant conditions, such as hydatidiform moles and choriocarcinoma (CH) (10). Despite improvements in diagnosis and treatment of these conditions, they can still lead to the illness and mortality of mother and child. Hence, it is imperative to find effective prevention and treatment measures for pregnancy-related events from the perspective of trophoblast invasion.

MicroRNAs (miRNA/miRs) are a class of noncoding RNAs 22 nucleotides in length that post-transcriptionally regulate diverse cellular processes by targeting mRNAs for cleavage or translational repression $(11,12)$. It has been suggested that miRNAs are involved in placental development and function (13). In addition, recent data have also indicated that miRNAs play a fundamental role in trophoblast proliferation, migration, invasion and apoptosis $(14,15)$. For example, 
it has been reported that miR-184 was highly expressed in recurrent spontaneous abortion (SA) and targeted zinc finger matrin-type 3 (ZMAT3) to promote the apoptosis of trophoblasts by upregulating Fas expression levels (16). miR-616-3p modulated cell proliferation and migration via targeting tissue factor pathway inhibitor 2 in PE (17). miR-21 was upregulated in hydatidiform mole tissues and promoted $\mathrm{CH}$ cell proliferation, migration and invasion (18). However, the association between miRNAs and trophoblast biological function in each of these previous studies was evaluated for a single event, such as PE or SA, thereby lacking a comprehensive analysis of such pregnancy-related events.

In the present study, samples were collected from multiple conditions with abnormal invasion capacity, and it was found that miR-181b-5p was highly expressed in events with impaired trophoblast invasiveness, such as SA (19) and PE $(20,21)$, but lowly expressed in $\mathrm{CH}$ which has excessive invasiveness $(10,22)$. These findings suggested that miR-181b-5p may have a pivotal role in multiple abnormal trophoblast invasion-related events involved in development or pathogenesis. Moreover, growing evidence has indicated that miR-181b-5p functions in regulating tumor cell migration and invasion $(23,24)$. Of note, it has been suggested that trophoblasts and tumor cells share similar biological characteristics, including proliferation, migration, invasion and apoptosis (25). Consequently, the vital role of miR-181b-5p in tumor cells is suggestive of its effect on the regulation of trophoblast cell migration and invasion. In the present study, bioinformatics analysis predicted that miR-181b-5p potentially targets the 3'-untranslated region (UTR) of sphingosine-1-phosphate (S1P) receptor 1 (S1PR1) mRNA; this was investigated via luciferase reporter assays. Therefore, the functional investigation of miR-181b-5p and its downstream targets in multiple abnormal trophoblast invasion-related events was the primary aim of the present study.

\section{Materials and methods}

Sample collection. Peripheral blood and tissue samples were obtained from 100 females at the Department of Obstetrics and Gynecology, Nanjing Maternity and Child Health Care Hospital from March 2013 to March 2018. Among these females, there were 40 healthy controls (20 first trimester, mean age, 30.9 \pm 5.2 years; 20 third trimester, mean age, $31.3 \pm 4.6$ years), and 20 cases of PE (mean age, $30.7 \pm 5.8$ years), $\mathrm{SA}$ (mean age, $32.6 \pm 3.6$ years) and $\mathrm{CH}$ (mean age, $32.4 \pm 3.2$ years), respectively. A total of $5 \mathrm{ml}$ blood was extracted from middle vein of the elbow of each participant. Red blood cells were removed by centrifugation at $1,880 \mathrm{x} \mathrm{g}$ for $20 \mathrm{~min}$ at $4^{\circ} \mathrm{C}$, and plasma was stored at $-20^{\circ} \mathrm{C}$ prior to use. Villi tissues were collected from normal early pregnancy (NV) and patients with SA, placenta samples were collected from normal full-term pregnancy (NP) and PE, and tumor tissue samples were collected from individuals with $\mathrm{CH}$. PE was defined as systolic blood pressure $\geq 140 \mathrm{~mm} \mathrm{Hg}$ and/or diastolic blood pressure $\geq 90 \mathrm{mmHg}$ on a minimum of two occasions at least 4-6 h apart, with urinary protein $\geq 0.3 \mathrm{~g} / 24 \mathrm{~h}$ after 20 weeks gestation. All women with $\mathrm{NV}$ terminated pregnancy for nonmedical reasons. Samples of SA were excluded if due to other causes, such as parental chromosomal, fetal chromosome abnormality, autoimmunity, thrombophilia, reproductive malformation, hormonal dysfunction (such as polycystic ovarian syndrome, thyroid abnormalities, diabetes mellitus, hyperprolactinemia or luteal insufficiency), folate deficiency or infection. All $\mathrm{CH}$ samples were collected after hysterectomy from patients without detectable infections or other systemic conditions. The clinical characteristics of these cases are shown in Tables I and II. The present study was approved by the Ethics Committee of the Nanjing Maternity and Child Health Care Hospital affiliated to Nanjing Medical University (Nanjing, China), and all enrolled patients provided written consent for sample collection and analyses.

Cell culture. HTR-8/SVneo cells, derived from primary first trimester human villous explant trophoblasts (26) and considered as a representative model of human extravillous trophoblasts (EVTs) (27), were selected as an in vitro model for the present study. HTR-8/SVneo cells were purchased from The Cell Bank of Type Culture Collection of the Chinese Academy of Sciences and were cultured in RPMI-1640 medium containing 10\% FBS (both Gibco; Thermo Fisher Scientific, Inc.), penicillin $(100 \mathrm{U} / \mathrm{ml})$ and streptomycin $(100 \mu \mathrm{g} / \mathrm{ml})$ at $37^{\circ} \mathrm{C}$ and $5 \% \mathrm{CO}_{2}$. Cells were passaged when they reached $90 \%$ confluence.

Oligonucleotide and plasmid transfection. Hsa-miR-181b-5p mimic and inhibitor, S1PR1 overexpression plasmid and their corresponding negative controls were purchased from Shanghai GenePharma Co., Ltd. The sequences were as follows: Has-miR-181b-5p mimics: 5'-AACAUUCAUUGC UGUCGGUGGGU-3' and 5'-ACCCACCGACAGCAAUGA AUGUU-3'; Has-miR-181b-5p mimics-NC: 5'-UUUGUA CUACACAAAAGUACUG-3' and 5'-CAGUACUUUUGU GUAGUACAAA-3'; Has-miR-181b-5p inhibitor: 5'-ACC CACCGACAGCAAUGAAUGUU-3'; and Has-miR-181b-5p inhibitor-NC: 5'-CAGUACUUUUGUGUAGUACAAA-3'. HTR-8/SVneo cells were seeded onto 6 -well plates at a density of $1 \times 10^{5}$ cells/well. When cells were $70-90 \%$ confluent, transient transfections were conducted using Lipofectamine ${ }^{\circledR}$ RNAi-MAX for oligonucleotides transfections or Lipofectamine 2000 for plasmid transfections (Thermo Fisher Scientific, Inc.), according to the manufacturer's instructions. The final concentration of all mimics, inhibitor and plasmid used for transfection was $100 \mathrm{nM}$. Transfection medium was replaced $8 \mathrm{~h}$ post-transfection and cells were harvested at $48 \mathrm{~h}$ for further experimentation. Transfection efficiency was examined via reverse transcription-quantitative PCR (RT-qPCR).

In vitro migration and invasion assays. An in vitro scratch assay was performed to evaluate cell migration ability. When cells reached $90 \%$ confluence, wounds were made by scraping confluent cell monolayers with a $100-\mu 1$ pipette tip, and non-adherent cells and debris were removed using PBS. In order to minimize cell proliferation, cells were cultured in serum-free medium. The width of the scratch was monitored every $12 \mathrm{~h}$, and the wound width was measured using a Nikon ECLIPSE Ts2 light microscope (Nikon Corporation; magnification, $\mathrm{x} 10$ ) at 0 and $48 \mathrm{~h}$, respectively. The percentage of wound closure areas was calculated using the following formula: (Wound width at $0 \mathrm{~h}$-wound width at $48 \mathrm{~h}$ )/wound 
Table I. Clinical characteristics of PE pregnancies and $\mathrm{CH}$.

\begin{tabular}{lccccc}
\hline Variable & $\mathrm{PE}(\mathrm{n}=20)$ & $\mathrm{NP}(\mathrm{n}=20)$ & $\mathrm{CH}(\mathrm{n}=20)$ & P-value (PE vs. NP) & P-value (CH vs. NP) \\
\hline Maternal age, years & $30.7 \pm 5.8$ & $31.3 \pm 4.6$ & $32.4 \pm 3.2$ & $>0.05$ & $>0.05$ \\
Week of pregnancy & $35.2 \pm 3.9$ & $38.5 \pm 2.1$ & After pregnancy & $>0.05$ & - \\
Birth weight, g & $2,412 \pm 872$ & $3,312 \pm 415$ & - & $<0.05$ & - \\
Systolic blood pressure, $\mathrm{mmHg}$ & $165 \pm 20.5$ & $111 \pm 10.4$ & $117 \pm 13.5$ & $<0.01$ & $>0.05$ \\
Diastolic blood pressure, $\mathrm{mmHg}$ & $109 \pm 16.1$ & $71 \pm 8.3$ & $76 \pm 6.4$ & $<0.01$ & $>0.05$ \\
Proteinuria, g/day & $5.23 \pm 1.34$ & $<0.3$ & $<0.3$ & $<0.01$ & $>0.05$
\end{tabular}

$\mathrm{NP}$, normal full-term pregnancy; $\mathrm{PE}$, preeclampsia; $\mathrm{CH}$, choriocarcinoma.

Table II. Clinical characteristics of SA and NV in the first trimester.

\begin{tabular}{lrrc}
\hline Variable & $\mathrm{SA}(\mathrm{n}=20)$ & $\mathrm{NV}(\mathrm{n}=20)$ & $\begin{array}{c}\text { P-value } \\
(\mathrm{SA} \text { vs. NV })\end{array}$ \\
\hline Maternal age, years & $32.6 \pm 3.6$ & $30.9 \pm 5.2$ & $>0.05$ \\
Week of pregnancy & $8.4 \pm 2.5$ & $9.1 \pm 1.3$ & $>0.05$ \\
\hline
\end{tabular}

SA, spontaneous abortion; NV, normal early pregnancy.

width at $0 \mathrm{~h} \times 100 \%$ using Image $\mathrm{J}$ software version 1.8 .0 (National Institutes of Health).

Invasion assays were performed using Transwell inserts (Costar; Corning, Inc.) pre-coated with Matrigel (BD Biosciences). Briefly, a total of $5 \times 10^{4}$ transfected cells suspended in $200 \mu 1$ RPMI-1640 medium without FBS were seeded in the upper compartment of Transwell inserts. As a chemoattractant, medium containing $20 \%$ FBS was added to the lower well. After a $24-\mathrm{h}$ incubation at $37^{\circ} \mathrm{C}$ and $5 \% \mathrm{CO}_{2}$, the cells remaining in the top side of the insert membrane (non-invaded cells) were gently cleared with cotton swabs and the cells invading to the bottom side of the insert membrane were fixed using $4 \%$ paraformaldehyde for $30 \mathrm{~min}$ at room temperature. After washing with PBS, the fixed cells were stained using $0.1 \%$ crystal violet for $30 \mathrm{~min}$ at room temperature, and the number of stained cells was counted using Image J software version 1.8.0 (National Institutes of Health) under a light microscope (Olympus Corporation, magnification, x10). A total of five random field views were selected for counting.

$R T-q P C R$. Total RNA was extracted from plasma, tissue and cells using TRIzol ${ }^{\circledR}$ reagent (Thermo Fisher Scientific, Inc.) according to the manufacturer's instructions. Then, the RNA was reverse-transcribed into cDNA using Reverse Transcription kit (Thermo Fisher Scientific, Inc.) according to the manufacturer's instructions. RT-qPCR was performed using a SYBR Premix Ex Taq ${ }^{\mathrm{TM}}$ II (Perfect Real-Time) kit (Takara Bio, Inc.) and Mastercycler ${ }^{\circledR}$ ep qrealplex thermal cycler (Eppendorf) as previously described $(28,29)$. The thermocycling conditions were as follows: Pre-degeneration at $95^{\circ} \mathrm{C}$ for $15 \mathrm{~min}$, followed by 40 cycles at $95^{\circ} \mathrm{C}$ for $5 \mathrm{sec}$, and annealing at $60^{\circ} \mathrm{C}$ for $30 \mathrm{sec}$. The highly conserved and universally expressed small nuclear RNA U6 was used as an endogenous control to normalize the miRNA RT-qPCR data. $\beta$-actin expression was used for the normalization of S1PR1 expression. All primers were synthesized by Thermo Fisher Scientific, Inc. The following primers were used: S1PR1 forward, 5'-CAGCAAATCGGACAATTCCT-3' and reverse, 5'-GCCAGCGACCAAGTAAAGAG-3'; $\beta$-actin forward, 5'-CCTGGCACCCAGCACAAT-3' and reverse, 5'-GGGCCG GACTCGTCATAC-3'; CXCL3: Forward, 5'-TCCGTGGTC ACTGAACTGC-3' and reverse, 5'-AGTTGGTGCTCCCCT TGTTC-3'; PPP3R1: Forward, 5'-TGGAAGAGTTCATGT CTCTGCCTGA-3' and reverse, 5'-TGACACTGAACTGAG AGACGCCCT-3'; miR-181b-5p: Forward, 5'-CCAGCTGGG CTCACTGAACAATGA-3' and reverse, 5'-CAACTGGTG TCGTGGAGTCGGC-3'; and U6: Forward, 5'-CTCGCTTCG GCAGCACAT-3' and reverse, 5'-AACGCTTCACGAATT TGCGT-3'. RT-qPCR data were analyzed using the $2^{-\Delta \Delta C q}$ method (30).

Luciferase assays. TargetScan version 7.2 (targetscan.org) and miRanda (version mirSVR; microrna.org/microrna/home.do) were used to predict the target gene of miR-181b-5p. The 3'-UTR region of S1PR1 and a seven-nucleotide mutated sequence located at 1,019-1,026 bp were cloned into a pmiR plasmid (Thermo Fisher Scientific, Inc.) to generate the recombinant constructs, pmiR-S1PR1-wild-type (WT) and pmiR-S1PR1-mutated (MUT), respectively. For the luciferase assay, HTR-8/SVneo cells were seeded onto 12-well plates at a density of $1 \times 10^{5}$ cells/well and co-transfected with $1 \mu \mathrm{g}$ pmiR-S1PR1-WT or pmiR-S1PR1-MUT and $1 \mu \mathrm{g}$ miR-181b-5p mimics or mimics control using Lipofectamine 2000 (Thermo Fisher Scientific, Inc.). Cells were cultured at $37^{\circ} \mathrm{C}$ and harvested after $24 \mathrm{~h}$ and Renilla and firefly luciferase activity was analyzed using a Dual-luciferase Reporter assay system (Promega Corporation). Renilla luciferase activity was normalized to firefly luciferase activity.

Western blotting. Total protein was extracted from cells and tissue using RIPA lysis buffer (Beyotime Institute of Biotechnology) supplemented with protease inhibitors (Beyotime Institute of Biotechnology). A BCA kit (Thermo Fisher Scientific, Inc.) was used to detect the protein concentration. Protein samples (30 $\mu \mathrm{g} / \mathrm{sample})$ were separated via $10 \%$ SDS-PAGE and then transferred to PVDF membranes. After blocking with 5\% non-fat dry milk at room temperature 

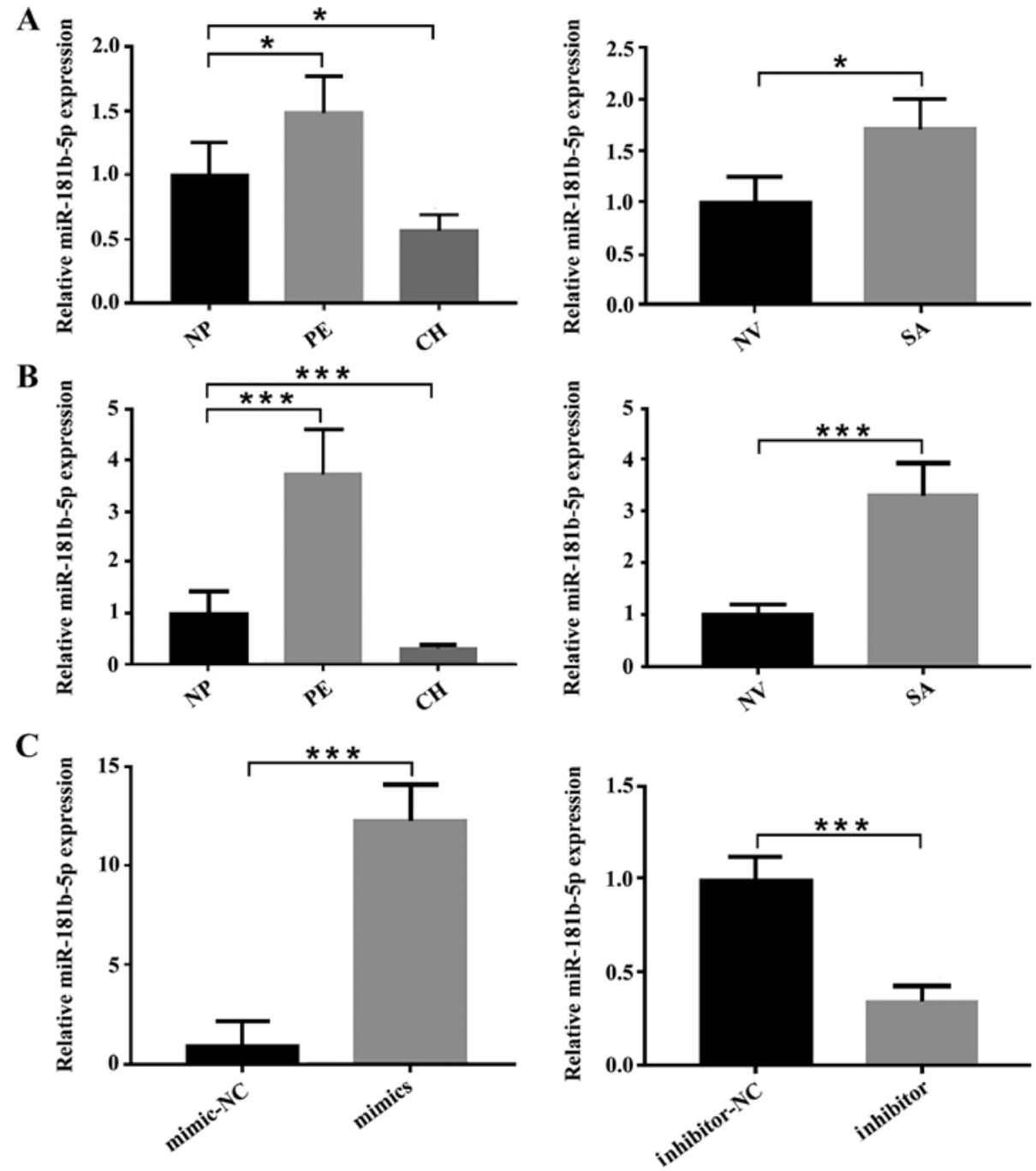

Figure 1. Expression levels of miR-181b-5p change with the invasiveness of pregnancy-related events. Differential miR-181b-5p expression in (A) plasma samples and (B) tissues samples of NP, PE, CH, NV, and SA were determined by RT-qPCR. (C) Overexpression and inhibition of miR-181b-5p in HTR-8/SVneo cells was demonstrated by RT-qPCR. The results are presented as the mean \pm SD of at least three experimental repeats. ${ }^{*} \mathrm{P}<0.05,{ }^{* * * *} \mathrm{P}<0.001 . \mathrm{NP}, \mathrm{normal}$ full-term pregnancy; PE, preeclampsia; $\mathrm{CH}$, choriocarcinoma; NV, normal early pregnancy; SA, spontaneous abortion; miR, microRNA; RT-qPCR, reverse transcription-quantitative PCR.

for $1 \mathrm{~h}$, the membranes were incubated overnight $\left(4^{\circ} \mathrm{C}\right)$ with primary antibodies: S1PR1 (1:1,000; cat. no. ab77076; Abcam), matrix metalloproteinase (MMP)-2 (1:1,000; cat. no. AF5330; Affinity Biosciences), MMP-9 (1:1,000; cat. no. AF5228; Affinity Biosciences), GAPDH (1:10,000; cat. no. ab9485; Abcam) and $\beta$-actin (1:5,000; cat. no. 60008-1-Ig; ProteinTech Group, Inc.). After three washes with TBS containing $0.1 \%$ Tween-20, the membranes were incubated with horseradish peroxidase-conjugated secondary antibodies (goat anti-rabbit; 1:5,000; cat. no. ab6721; Abcam) for $1 \mathrm{~h}$ at room temperature. Specific immunoreactive protein bands were visualized using an enhanced chemiluminescence protein detection kit (EMD Millipore). The quantification of the western blotting results was performed using ImageJ software version 1.8.0 (National Institutes of Health).

Statistical analysis. All data were analyzed using SPSS 17.0 (SPSS, Inc.) and presented as the mean \pm SD based on at least three independent experiments. Unpaired Student's t-test was used to perform comparisons of two groups, and Dunnett's test was used following one-way ANOVA to assess multiple comparisons. $\mathrm{P}<0.05$ was considered to indicate a statistically significant difference.

\section{Results}

miR-181b-5p is upregulated in low invasive event samples and downregulated in highly invasive event samples. To explore the function of miR-181b-5p in the events caused by abnormal trophoblast invasion, plasma and tissue samples were collected from multiple conditions with abnormal invasion capacity, including SA (impaired trophoblast invasiveness), $\mathrm{PE}$ (impaired trophoblast invasiveness) and $\mathrm{CH}$ (excessive invasiveness). The miR-181b-5p expression of the samples was detected using RT-qPCR. These results demonstrated that circulating miR-181b-5p was significantly upregulated in the SA and PE groups, but downregulated in the $\mathrm{CH}$ group, compared with the respective controls (Fig. 1A). The results using tissue samples were consistent with the plasma samples (Fig. 1B). These findings suggested the potential importance 
A
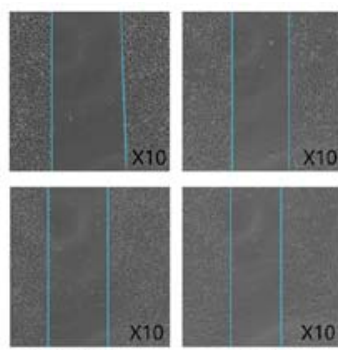

mimic-NC

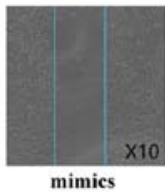

mimics

B
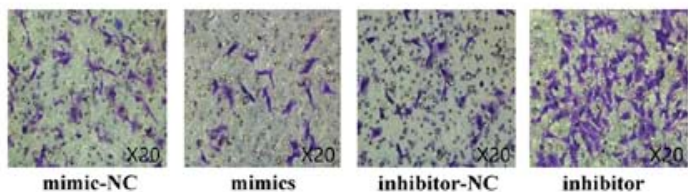
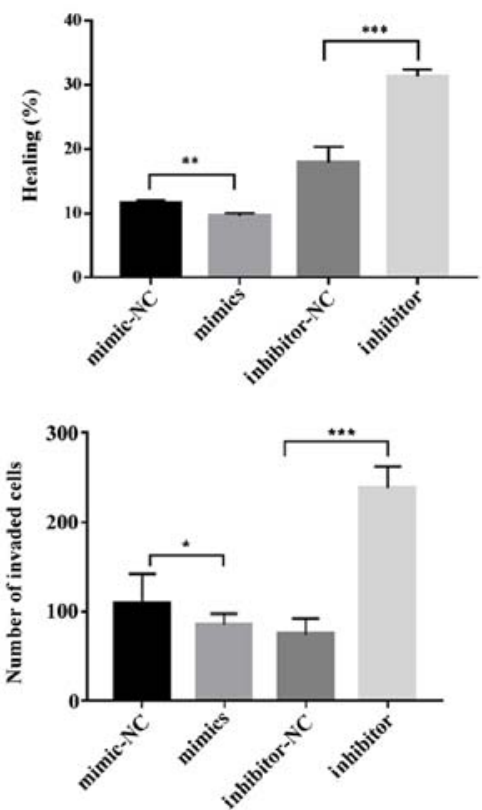
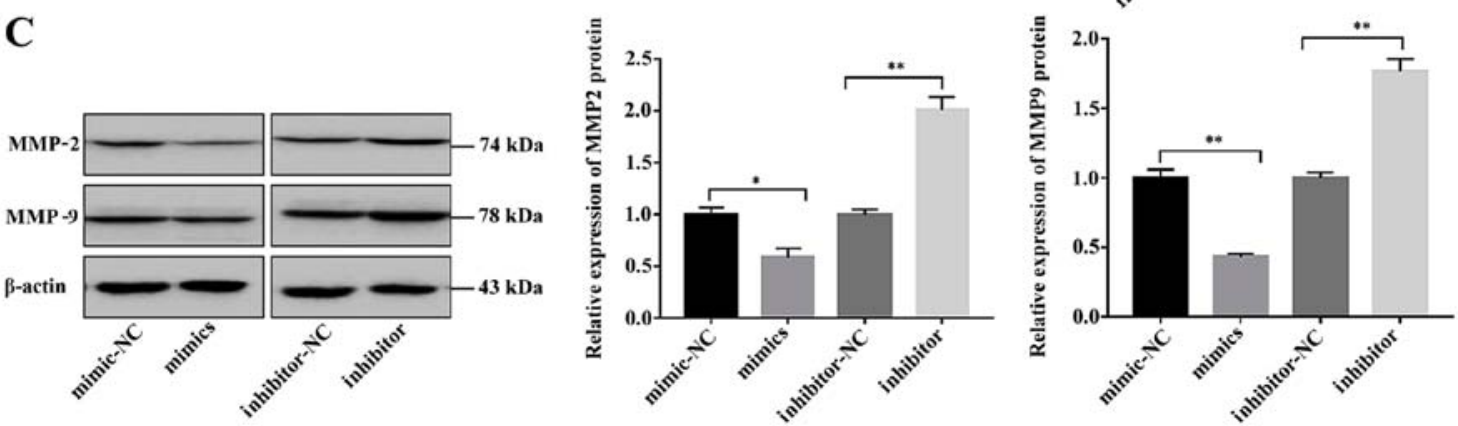

Figure 2. Effects of transient overexpression or knockdown of miR-181b-5p on the migratory and invasive abilities of HTR-8/SVneo cells. (A) HTR-8/SVneo cell migration was inhibited upon transfection with miR-181b-5p mimics and enhanced upon transfection with miR-181b-5p inhibitor. (B) Transfected cells were assessed by Transwell assays. HTR-8/SVneo cell invasion was inhibited upon transfection with miR-181b-5p mimics and enhanced upon transfection with miR-181b-5p inhibitor. (C) Western blotting analysis showed that the expression levels of MMP-2 and MMP-9 were significantly decreased in miR-181b-5p mimics-treated cells and increased in miR-181b-5p inhibitor-treated cells compared with corresponding controls. The results are presented as the mean \pm SD of at least three experimental repeats. ${ }^{*} \mathrm{P}<0.05,{ }^{* *} \mathrm{P}<0.01,{ }^{* * *} \mathrm{P}<0.001$. MMP, matrix metalloproteinase; NC, negative control; miR, microRNA.

of miR-181b-5p in the pathogenesis of these events caused by abnormal trophoblast invasion.

To further evaluate the biological functions of miR-181b-5p in events with abnormal trophoblast invasion, up- or downregulated miR-181b-5p expression levels were induced in HTR-8/SVneo cells by transfection with miR-181b-5p mimics or inhibitors, respectively (Fig. 1C).

miR-181b-5p reduces migration and invasion of HTR-8/SVneo cells. Wound healing and Transwell assays were conducted to explore the roles of miR-181b-5p in trophoblast cell migration and invasion. As shown in Fig. 2A, overexpression of miR-181b-5p suppressed HTR-8/SVneo cell migration, while downregulation of miR-181b-5p significantly promoted HTR-8/SVneo cell migration. Similar results were observed in the Transwell assay experiments (Fig. 2B); as hypothesized, miR-181b-5p overexpression inhibited, and knockdown of miR-181b-5p significantly increased, the invasion of HTR-8/SVneo cells. Furthermore, to investigate the effect of miR-181b-5p on the invasion-related enzymes, MMP-2 and MMP-9, in HTR-8/SVneo cells, western blotting was performed. At different stages of embryo implantation, trophoblast cells secrete different quantities and types of MMP, among which MMP-2 and MMP-9 are the most important enzymes involved in trophoblastic invasion (31). It was revealed that the expression of MMP-2 and MMP-9 was decreased by miR-181b-5p mimics treatment, whilst MMP-2 and MMP-9 expression was increased by miR-181b-5p inhibitor treatment (Fig. 2C). Collectively, these results demonstrated that miR-181b-5p could suppress the migratory and invasive abilities of trophoblasts.

SIPRI is a direct target of miR-181b-5p. To investigate the molecular mechanisms via which miR-181b-5p suppressed the invasion and migration of trophoblasts, the potential targets of miR-181b-5p were searched for using the miRNA target prediction tools TargetScan and miRanda. miR-181b-5p was found to target the 3'-UTR region of three genes: S1PR1, $\mathrm{C}-\mathrm{X}-\mathrm{C}$ motif chemokine ligand 3 and protein phosphatase 3 regulatory subunit $\mathrm{B}, \alpha$. Further RT-qPCR analysis suggested that, of these three, S1PR1 mRNA was most markedly affected by $\mathrm{miR}-181 \mathrm{~b}-5 \mathrm{p}$ overexpression in trophoblast cells (data not shown); therefore, S1PR1 was selected for further experimentation. Fig. 3A showed the S1PR1 sequence complementary to 
A

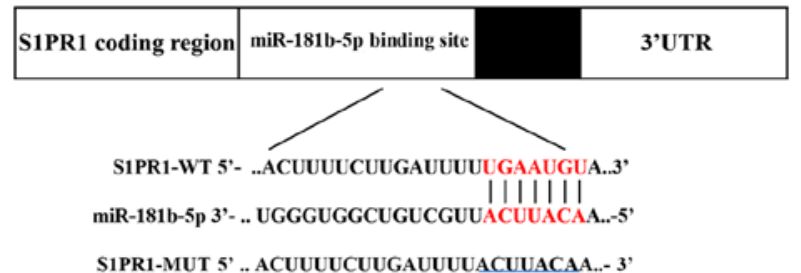

C

D
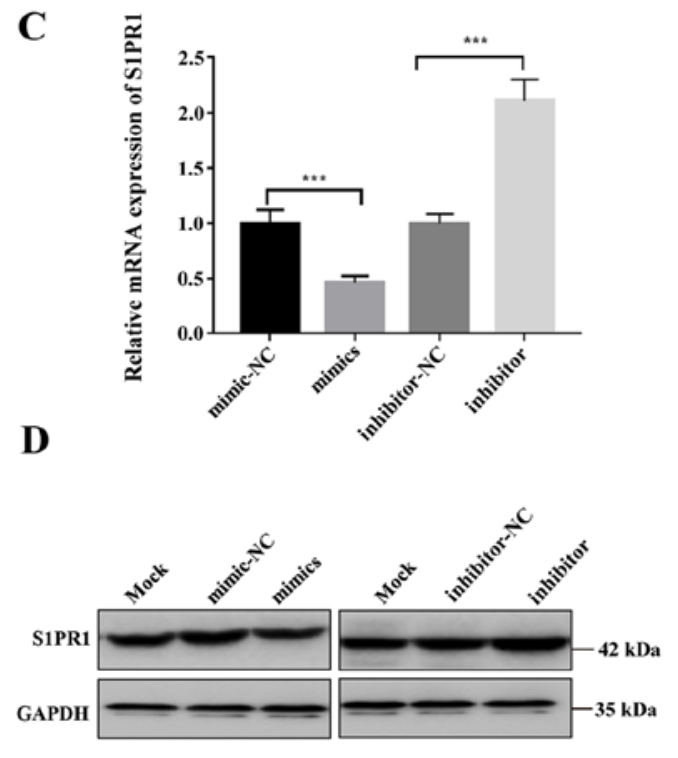
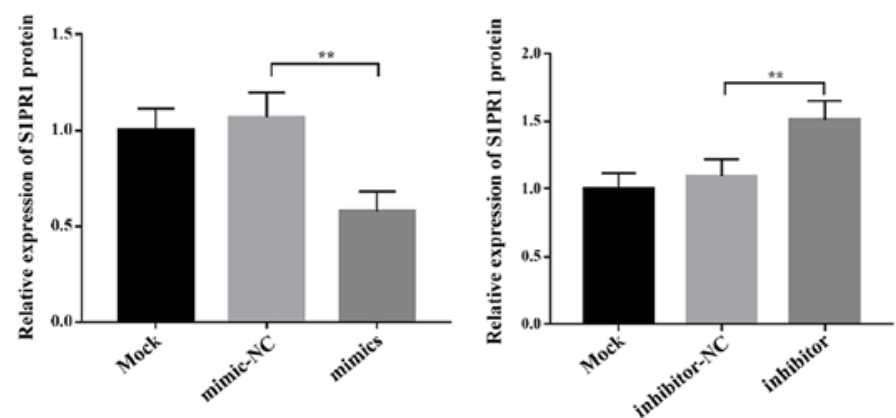

Figure 3. S1PR1 is a direct target of miR-181b-5p. (A) Sequence alignment via bioinformatics analysis between miR-181b-5p and its putative binding site in the S1PR1 3'-UTR. (B) Luciferase activities of reporter vectors containing either the WT or the MUT S1PR1 3'-UTR were measured in the presence of miR-181b-5p mimic or mimic-NC. ${ }^{* * *} \mathrm{P}<0.001$. (C) Relative S1PR1 mRNA expression in HTR-8/SVneo cells after being transfected with miR-181b-5p mimics, mimic-NC, miR-181b-5p inhibitor or inhibitor-NC. (D) S1PR1 relative protein expression in HTR-8/SVneo cells after being transfected with miR-181b-5p mimics, mimic-NC, miR-181b-5p inhibitor or inhibitor-NC. The results are presented as the mean $\pm \mathrm{SD}$ of at least three experimental repeats. ${ }^{* *}<0.01$, ${ }^{* * *} \mathrm{P}<0.001$. WT, wild-type; MUT, mutated; NC, negative control; UTR, untranslated region; miR, microRNA; S1PR1, sphingosine-1-phosphate receptor 1.

miR-181b-5p. In order to confirm that S1PR1 might be a direct target of miR-181b-5p, luciferase assays were performed by co-transfection of pmiR-S1PR1 luciferase reporter plasmids and miR-181b-5p mimics. The results showed that transfection with miR-181b-5p mimics significantly reduced luciferase activity of S1PR1-WT, but had no effect on the S1PR1-MUT (Fig. 3B). RT-qPCR and western blotting further validated that S1PR1 was a target of miR-181b-5p: S1PR1 mRNA and protein expression in trophoblasts were both decreased after administration of miR-181b-5p mimics and upregulated after transfection with miR-181b-5p inhibitor (Fig. 3C and D). Taken together, these results strongly indicated that miR-181b-5p directly and specifically binds to the target site in the 3'-UTR of S1PR1.

Expression of S1PRI in abnormal trophoblast invasion-related events. To evaluate whether the expression of S1PR1 is associated with miR-181b-5p expression in diseased states, mRNA and protein expression levels of S1PR1 were examined by RT-qPCR and western blotting in tissues from multiple conditions with abnormal trophoblast invasion. As compared with normal placenta or normal villus tissues, S1PR1 mRNA and protein expression was found to be significantly downregulated in the PE and SA groups, but upregulated in the $\mathrm{CH}$ group (Fig. 4A and B). This suggested that miR-181b-5p expression was negatively associated with S1PR1 expression in tissue samples from normal pregnancies and abnormal trophoblast invasion-related events.

Overexpression of SIPRI attenuates the effects induced by miR-181b-5p in trophoblast cells. To determine whether the suppressive effect of miR-181b-5p on trophoblast invasion was mediated by S1PR1 repression, S1PR1 was overexpressed in HTR-8/SVneo cells. As shown in Fig. S1, the expression of S1PR1 mRNA was significantly upregulated after plasmid transfection. Subsequently, miR-181b-5p was overexpressed together with S1PR1 in HTR-8/SVneo cells to perform a rescue experiment. Of note, the migration and invasion assay results showed that restoring expression of S1PR1 counteracted the inhibitory effects of miR-181b-5p overexpression on the migration and invasion of the trophoblast cells (Fig. 5A and B). These data indicated that miR-181b-5p suppressed trophoblast migration and invasion at least partially by inhibiting S1PR1.

\section{Discussion}

Trophoblast invasion has been one of the major foci of placental research due to its pivotal role in the process of embryo implantation and placentation. A number of similarities of 

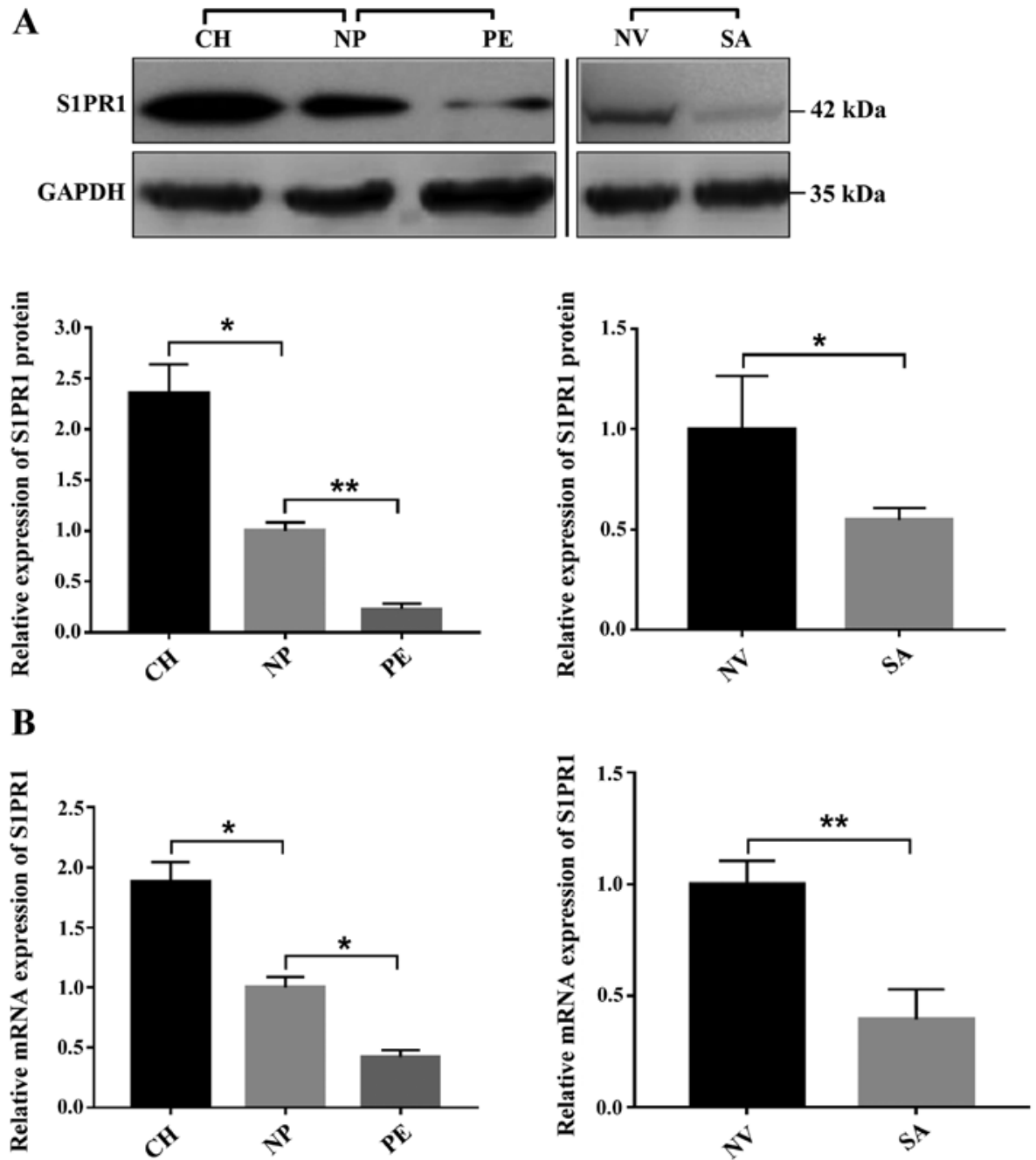

Figure 4. mRNA and protein expression levels of S1PR1 in tissue samples. (A) Composite image shows gels with tracks from different exposures. Compared with NP, S1PR1 protein levels in the PE group were found to be significantly downregulated, but those in the CH group were upregulated. Compared with NV tissues, S1PR1 protein levels in the SA group were found to be significantly downregulated. Bands were semi-quantitatively compared between groups. (B) Relative mRNA expression of S1PR1 in the peripheral blood samples of normal pregnant women and women with abnormal trophoblast invasion-related events. The results are presented as the mean $\pm \mathrm{SD}$ of at least three experimental repeats. ${ }^{*} \mathrm{P}<0.05,{ }^{* * *} \mathrm{P}<0.01$. NP, normal full-term pregnancy; $\mathrm{PE}$, preeclampsia; $\mathrm{CH}$, choriocarcinoma; NV, normal early pregnancy; SA, spontaneous abortion; S1PR1, sphingosine-1-phosphate receptor 1.

invasion behavior exist between trophoblasts and cancer cells (25). However, trophoblast invasion is transient with rigorous spatial and temporal confines (32). Multiple studies have verified that disorders of trophoblast invasion could trigger a series of pregnancy-related events at various stages of pregnancy: Abnormalities in vascular invasion of trophoblast in non-arterial vessels, such as veins and lymphatic vessels, were observed in the early miscarriage (6); PE was found to derive from a defect in trophoblast invasion that resulted in impaired uterine spiral artery remodeling in the second or third trimester $(33,34)$; and abnormally extensive invasion of trophoblasts was suggested to lead to premalignant or malignant conditions, such as $\mathrm{CH}$ after pregnancy $(10,22)$. miRNAs have emerged as crucial regulators that mediate cellular activities, including invasion (35). Accumulating evidence has suggested that miRNAs are associated with placental formation and embryonic development by affecting the biological function of trophoblasts (36). However, most prior studies evaluated the association between miRNAs and trophoblasts' biological function only in a single event. In the present study, samples were collected from multiple events with abnormal invasion capacity and miR-181b-5p expression was examined in each group of events. The results showed that miR-181b-5p was decreased in both plasma and tissue samples of $\mathrm{CH}$ (excessive invasiveness), but elevated in SA and PE (impaired invasiveness), which suggested an important role for miR-181b-5p in trophoblast invasion-related adverse pregnancy outcomes.

miR-181b-5pisamemberofthemiR-181 family (miR-181a-d). Functional studies have indicated that miR-181b-5p may affect malignant cell migration and invasion $(23,37)$. For example, miR-181b suppresses invasion and migration of glioma cells by targeting NOVA alternative splicing regulator 1 (23). Furthermore, in the present study, miR-181b-5p expression was observed in the trophoblast cell line HTR-8/SVneo, which is similar to EVT cells (27). Wound healing and Transwell assays demonstrated that pretreatment with miR-181b-5p mimic or inhibitor suppressed or promoted, respectively, the invasion and migration of HTR-8/SVneo cells. These results were further verified by examining invasion-related proteins MMP-2 and MMP-9. Based on these results, it was indicated 
A
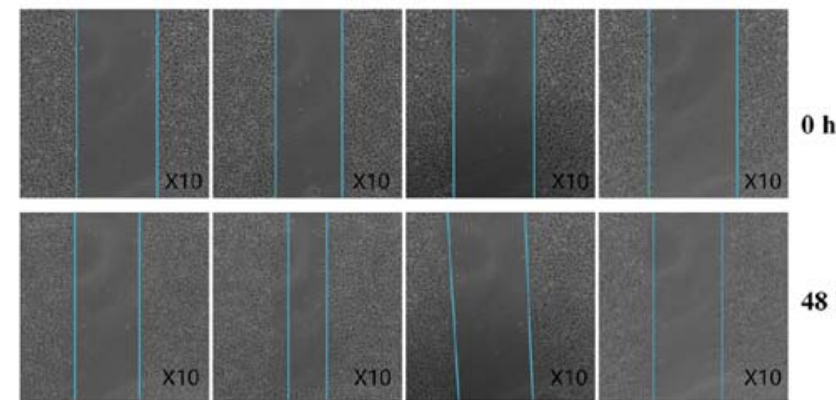

SIPR1-NC

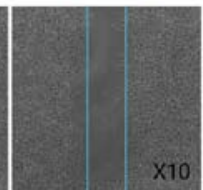

SIPR1

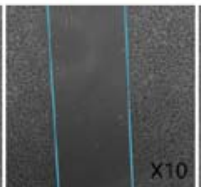

mimics-miR$181 \mathrm{~b}+$ S1PR1-NC

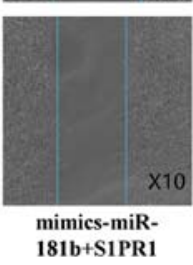

$48 \mathrm{~h}$

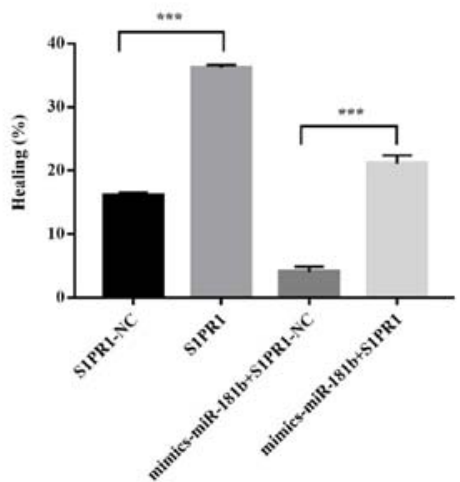

B

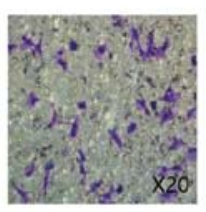

S1PR1-NC

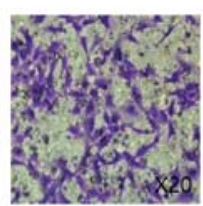

S1PR1

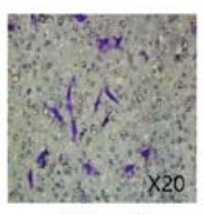

mimics-miR181b+S1PR1-NC
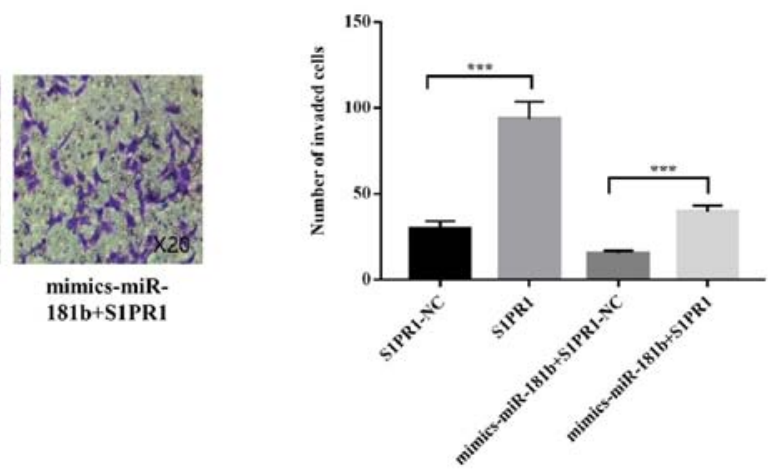

Figure 5. Overexpression of S1PR1 reverses the effects of miR-181b-5p overexpression. (A) Cell migration in HTR-8/SVneo cells transfected with miR-181b-5p mimics, or co-transfected with miR-181b-5p mimics and S1PR1 overexpression plasmids was determined by wound healing assays. (B) Cell invasion in HTR-8/SVneo cells transfected with miR-181b-5p mimics, or co-transfected with miR-181b-5p mimics and S1PR1 overexpression plasmids was determined by Transwell assays. The results are presented as the mean $\pm \mathrm{SD}$ of at least three experimental repeats. ${ }^{* * *} \mathrm{P}<0.001$. NC, negative control; miR, microRNA; S1PR1, sphingosine-1-phosphate receptor 1.

that miR-181b-5p may suppress trophoblast invasion by inhibiting the levels of MMP-2 and MMP-9.

In the present study, S1PR1 was identified as a candidate target gene of miR-181b-5p through two publicly available algorithms, TargetScan and miRanda. Luciferase reporter assays indicated that miR-181b-5p could inhibit S1PR1 expression by directly binding to the 3'-UTR of S1PR1, which was validated by RT-qPCR and western blotting. In addition, further rescue experiments suggested that overexpression of S1PR1 weakened the inhibitory effects of miR-181b-5p on trophoblast migration and invasion. S1PR1, also known as endothelial differentiation gene 1 , is a $G$ protein-coupled receptor implicated in the regulation of vascular remodeling, endothelial barrier integrity and vascular tone (38). Uterine spiral artery remodeling and trophoblast invasion are important for increasing uteroplacental blood flow and lowering maternal vascular resistance in pregnancy (39). It is widely accepted that successful placentation depends on the proper invasion of EVT cells into maternal tissues. Previous studies demonstrated that S1PR1 is expressed in human EVT cells (40), and that S1P could regulate migration and invasion of the trophoblast cells via S1P receptors $(41,42)$. Evidence suggests that S1P/S1PR1 axis activation causes induction of MMP-2 and MMP-9, and thus enhances invasion and migration $(42,43)$. In the present study, it was reported for the first time that the invasion-inhibiting effect of miR-181b-5p is mediated at least partially by suppressing S1PR1. HTR-8/SVneo cells were selected due to their similarity to EVT cells. In future studies, the in vitro functional role of miR-181b-5p should be validated in primary cells, which are a closer model of the physiological conditions. In addition, the data presented in the present study were mainly obtained from human trophoblast cell lines in vitro; therefore, in future experiments, in vivo models should be used to explore miR-181b-5p function and its underlying mechanism in the pathogenesis of multiple abnormal trophoblast invasion-associated events.

In summary, to the authors' best knowledge, the present study provides the first evidence that dysregulated miR-181b-5p contributes to pregnancy-related events by affecting the migration and invasion of trophoblast cells via direct targeting of S1PR1.

\section{Acknowledgements}

Not applicable.

\section{Funding}

This study was supported by the National Natural Science Foundation of China (grant no. 81501252), the Natural Science Foundation of Jiangsu Province (grant no. BK20140084) and the Medical Science and Technology Development Foundation of Nanjing Department of Health (Grant No. YKK15162).

\section{Availability of data and materials}

The datasets used and/or analyzed during the current study are available from the corresponding author on reasonable request. 


\section{Authors' contributions}

JM, YZ and LX conceived the study and performed the experiments. JM drafted the manuscript and performed the analysis. XH and XZ contributed to drafting the manuscript, interpreting data and coordinating the study. XZ provided the funding. All authors read and approved the final manuscript.

\section{Ethics approval and consent to participate}

The present study was approved by the Ethics Committee of the Nanjing Maternity and Child Health Care Hospital affiliated to Nanjing Medical University. All enrolled patients provided written consent for participation in the study, sample collection and analyses.

\section{Patient consent for publication}

Not applicable.

\section{Competing interests}

The authors declare that they have no competing interests.

\section{References}

1. Norwitz ER, Schust DJ and Fisher SJ: Implantation and the survival of early pregnancy. N Engl J Med 345: 1400-1408, 2001.

2. Knöfler M and Pollheimer J: Human placental trophoblast invasion and differentiation: A particular focus on Wnt signaling. Front Genet 4: 190, 2013.

3. Soundararajan R and Rao AJ: Trophoblast 'pseudo-tumorigenesis': Significance and contributory factors. Reprod Biol Endocrinol 2: 15, 2004.

4. Okae H, Toh H, Sato T, Hiura H, Takahashi S, Shirane K, Kabayama Y, Suyama M, Sasaki H and Arima T: Derivation of human trophoblast stem cells. Cell Stem Cell 22: 50-63.e56, 2018.

5. Norwitz ER: Defective implantation and placentation: Laying the blueprint for pregnancy complications. Reprod Biomed Online 13: 591-9, 2006

6. Windsperger K, Dekan S, Pils S, Golletz C, Kunihs V, Fiala C, Kristiansen G, Knöfler M and Pollheimer J: Extravillous trophoblast invasion of venous as well as lymphatic vessels is altered in idiopathic, recurrent, spontaneous abortions. Hum Reprod 32: 1208-1217, 2017.

7. Ye Y, Vattai A, Zhang X, Zhu J, Thaler CJ, Mahner S, Jeschke U and von Schönfeldt V: Role of Plasminogen activator inhibitor type 1 in pathologies of female reproductive diseases. Int $\mathrm{J}$ Mol Sci 18: 1651,2017.

8. Ding J, Cheng Y, Zhang Y, Liao S, Yin T and Yang J: The miR-27a-3p/USP25 axis participates in the pathogenesis of recurrent miscarriage by inhibiting trophoblast migration and invasion. J Cell Physiol 234: 19951-19963, 2019.

9. Cudihy D and Lee RV: The pathophysiology of pre-eclampsia: Current clinical concepts. J Obstet Gynaecol 29: 576-582, 2009.

10. Li HW, Tsao SW and Cheung AN: Current understandings of the molecular genetics of gestational trophoblastic diseases. Placenta 23: 20-31, 2002.

11. Bartel DP: MicroRNAs: Genomics, biogenesis, mechanism, and function. Cell 116: 281-297, 2004

12. Sun W, Julie Li YS, Huang HD, Shyy JY and Chien S: microRNA: A master regulator of cellular processes for bioengineering systems. Annu Rev Biomed Eng 12: 1-27, 2010.

13. Sadovsky Y, Mouillet JF, Ouyang Y, Bayer A and Coyne CB: The function of trophomirs and other MicroRNAs in the human placenta. Cold Spring Harb Perspect Med 5: a023036, 2015.

14. Vaiman D: Genes, epigenetics and miRNA regulation in the placenta. Placenta 52: 127-133, 2017.

15. Cai M, Kolluru GK and Ahmed A: Small molecule, big prospects: MicroRNA in pregnancy and its complications. J Pregnancy 2017: 6972732, 2017.
16. Zhang Y, Zhou J, Li MQ, Xu J, Zhang JP and Jin LP: MicroRNA-184 promotes apoptosis of trophoblast cells via targeting WIG1 and induces early spontaneous abortion. Cell Death Dis 10: 223, 2019

17. Xu Y, Wu D, Jiang Z, Zhang Y, Wang S, Ma Z, Hui B, Wang J, Qian W, Ge Z and Sun L: MiR-616-3p modulates cell proliferation and migration through targeting tissue factor pathway inhibitor 2 in preeclampsia. Cell Prolif 51: e12490, 2018.

18. Wang YX, Zhao JR, Xu YY, Wu WB and Zhang HJ: miR-21 is overexpressed in hydatidiform mole tissues and promotes proliferation, migration, and invasion in choriocarcinoma cells. Int $\mathrm{J}$ Gynecol Cancer 27: 364-374, 2017.

19. Ball E, Bulmer JN, Ayis S, Lyall F and Robson SC: Late sporadic miscarriage is associated with abnormalities in spiral artery transformation and trophoblast invasion. J Pathol 208: 535-542, 2006.

20. Fisher SJ: Why is placentation abnormal in preeclampsia? Am J Obstet Gynecol 213 (Suppl 4): S115-S122, 2015.

21. Goldman-Wohl D and Yagel S: Regulation of trophoblast invasion: From normal implantation to pre-eclampsia. Mol Cell Endocrinol 187: 233-238, 2002.

22. Hiden U, Bilban M, Knöfler M and Desoye G: Kisspeptins and the placenta: Regulation of trophoblast invasion. Rev Endocr Metab Disord 8: 31-39, 2007.

23. Zhi F, Wang Q, Deng D, Shao N, Wang R, Xue L, Wang S, Xia X and Yang Y: MiR-181b-5p downregulates NOVA1 to suppress proliferation, migration and invasion and promote apoptosis in astrocytoma. PLoS One 9: e109124, 2014.

24. Wang X, Chen X, Meng Q, Jing H, Lu H, Yang Y, Cai L and Zhao Y: MiR-181b regulates cisplatin chemosensitivity and metastasis by targeting TGF $\beta$ R $1 /$ Smad signaling pathway in NSCLC. Sci Rep 5: 17618, 2015.

25. Lala PK, Lee BP, Xu G and Chakraborty C: Human placental trophoblast as an in vitro model for tumor progression. Can J Physiol Pharmacol 80: 142-149, 2002.

26. Irving JA, Lysiak JJ, Graham CH, Hearn S, Han VK and Lala PK: Characteristics of trophoblast cells migrating from first trimester chorionic villus explants and propagated in culture. Placenta 16 : 413-433, 1995.

27. Graham CH,Hawley TS, Hawley RG, MacDougall JR, Kerbel RS, Khoo N and Lala PK: Establishment and characterization of first trimester human trophoblast cells with extended lifespan. Exp Cell Res 206: 204-211, 1993.

28. Chen C, Ridzon DA, Broomer AJ, Zhou Z, Lee DH, Nguyen JT, Barbisin M, Xu NL, Mahuvakar VR, Andersen MR, et al: Real-time quantification of microRNAs by stem-loop RT-PCR. Nucleic Acids Res 33: e179, 2005.

29. Varkonyi-Gasic E, Wu R, Wood M, Walton EF and Hellens RP: Protocol: A highly sensitive RT-PCR method for detection and quantification of microRNAs. Plant Methods 3: 12, 2007.

30. Livak KJ and Schmittgen TD: Analysis of relative gene expression data using real-time quantitative PCR and the 2(-Delta Delta C(T)) method. Methods 25: 402-408, 2001

31. Chen L, Nakai M, Belton RJ Jr and Nowak RA: Expression of extracellular matrix metalloproteinase inducer and matrix metalloproteinases during mouse embryonic development. Reproduction 133: 405-414, 2007.

32. van den Brûle F, Berndt S, Simon N, Coulon C, Le Goarant J, Munaut C, Noël A, Frankenne F and Foidart JM: Trophoblast invasion and placentation: Molecular mechanisms and regulation. Chem Immunol Allergy 88: 163-180, 2005.

33. Kaufmann P, Black S and Huppertz B: Endovascular trophoblast invasion: Implications for the pathogenesis of intrauterine growth retardation and preeclampsia. Biol Reprod 69: 1-7, 2003.

34. Powe CE, Levine RJ and Karumanchi SA: Preeclampsia, a disease of the maternal endothelium: The role of antiangiogenic factors and implications for later cardiovascular disease. Circulation 123: 2856-2869, 2011.

35. Kotlabova K, Doucha J and Hromadnikova I: Placental-specific microRNA in maternal circulation-identification of appropriate pregnancy-associated microRNAs with diagnostic potential. J Reprod Immunol 89: 185-191, 2011.

36. Navarro A and Monzo M: MicroRNAs in human embryonic and cancer stem cells. Yonsei Med J 51: 622-632, 2010.

37. He L, Yao H, Fan LH, Liu L, Qiu S, Li X, Gao JP and Hao CQ: MicroRNA-181b expression in prostate cancer tissues and its influence on the biological behavior of the prostate cancer cell line PC-3. Genet Mol Res 12: 1012-1021, 2013. 
38. Kitano T, Usui S, Takashima SI, Inoue O, Goten C, Nomura A Yoshioka K, Okajima M, Kaneko S, Takuwa Y and Takamura M: Sphigosine-1-phosphate receptor 1 promotes neointimal hyperplasia in a mouse model of carotid artery injury. Biochem Biophys Res Commun 511: 179-184, 2019.

39. Cui Y, Wang W, Dong N, Lou J, Srinivasan DK, Cheng W, Huang X, Liu M, Fang C, Peng J, et al: Role of corin in trophoblast invasion and uterine spiral artery remodelling in pregnancy. Nature 484: 246-250, 2012

40. Goyal P, Brünnert D, Ehrhardt J, Bredow M, Piccenini S and Zygmunt M: Cytokine IL-6 secretion by trophoblasts regulated via sphingosine-1-phosphate receptor 2 involving Rho/Rho-kinase and Rac1 signaling pathways. Mol Hum Reprod 19: 528-538, 2013.
41. Westwood M, Al-Saghir K, Finn-Sell S, Tan C, Cowley E, Berneau S, Adlam D and Johnstone ED: Vitamin D attenuates sphingosine-1-phosphate (S1P)-mediated inhibition of extravillous trophoblast migration. Placenta 60: 1-8, 2017.

42. Yang W, Li Q and Pan Z: Sphingosine-1-phosphate promotes extravillous trophoblast cell invasion by activating MEK/ERK/MMP-2 signaling pathways via S1P/S1PR1 axis activation. PLoS One 9: e106725, 2014.

43. Kim ES, Kim JS, Kim SG, Hwang S, Lee CH and Moon A: Sphingosine 1-phosphate regulates matrix metalloproteinase-9 expression and breast cell invasion through S1P3-Gaq coupling. J Cell Sci 124: 2220-2230, 2011. 